\title{
Reconstructing Polarisation Components from Unpolarised Images
}

\author{
Lin $\mathrm{Gu}^{\dagger}$ Cong Phuoc Huynh ${ }^{\dagger, *}$ Antonio Robles-Kelly ${ }^{\dagger, *}$ \\ ${ }^{\dagger}$ Research School of Engineering, Australian National University, Canberra ACT 0200, Australia \\ *NICTA , Locked Bag 8001, Canberra ACT 2601, Australia
}

\begin{abstract}
In this paper, we develop a method for reconstructing the polarisation components from unpolarised imagery. Our approach rests on a model of polarisation which accounts for reflection from rough surfaces illuminated at moderate and large angles of incidence. Departing from the microfacet structure of rough surfaces, we relate the maximal and minimal polarimetric intensities to the diffuse and specular components of an unpolarised image via the Fresnel reflection theory. This allows us to reconstruct the polarimetric components from a single unpolarised image. Thus, the model presented here provides a link between the microfacet structure and polarisation of light upon reflection from rough surfaces. We evaluate the accuracy of the reconstructed polarisation components and illustrate the utility of the method for the simulation of a polarising filter on real-world images.
\end{abstract}

\section{INTRODUCTION}

The polarisation of light is a property that describes the oscillations of its electric field in the plane perpendicular to the direction of propagation. The effects of polarisation can be captured by devices such as polarimeters and more recently, polarisation cameras [1]. Such developments have broadened the applications of polarisation to areas such as target detection [2] and material property recovery [3].

Indeed, polarisation has shown to be an effective tool in revealing the underlying properties of the surface under study. For instance, Wolff and Boult [3] made use of polarisation images to distinguish metallic from dielectric materials based on the ratio between the two Fresnel reflection components, $i$.e. the so-called Fresnel ratio. More recently, Chen and Wolff [4] examined the phase shift between the polarisation components reflected from surfaces to distinguish conducting metallic from dielectric materials.

A vast body of Computer Vision literature has been dedicated to the modelling of unpolarised reflectance for rough surfaces. Oren and Nayar [5] generalised the Lambertian model to account for variation of reflected light with respect to the viewing direction. In earlier work, Torrance and Sparrow [6] formulated a reflectance model based on the Fresnel reflection theory to explain the off-specular spike phenomenon, in which the specular spike occurred at a viewing angle larger than the specular reflection angle. Beckmann [7] modelled the surface reflectance as a wave scattering process. However, none of the latter models takes into account polarisation effects.

On the other hand, a number of polarimetric reflectance models have been formulated for smooth surfaces. In [8],

NICTA is funded by the Australian Government as represented by the Department of Broadband, Communications and the Digital Economy and the Australian Research Council through the ICT Centre of Excellence program.
Wolff derived a polarisation reflectance model based on the Fresnel reflection theory and employed it for the classification of dielectrics and metals. In addition, the Wolff diffuse reflectance model [9] could be extended to derive the parallel and perpendicular polarisation components with respect to the plane of reflection. We note that these models only apply to smooth surfaces without a notion of their level of roughness. In these models, polarisation is expressed as being dependent on the material refractive index and the reflection geometry in a single equation.

Although reflectance modelling is a significant area of research within physics-based computer vision, there has been little work on the modelling of polarisation of light reflected from rough surfaces. An early empirical approach was adopted by Torrance, Sparrow and Birkebak to measure the specular reflectance distribution of rough surfaces for different polarisation orientations [10]. In a separate development, Priest and Germer provided a physics-based polarimetric bidirectional reflectance distribution function (BRDF) of light scattered from micro-facets [11]. The model describes the most general polarisation state of light in terms of physical parameters including the refractive index and the surface roughness using Stokes vectors and the Mueller matrix. As a result, this model permits the estimation of refractive index and reflection angle from polarisation images [12].

Although being general in nature, the polarimetric model in [11] is often overly complex and cumbersome for practical applications. This is due to the many degrees of freedom inherent in the elements of the Mueller matrix that need to be measured precisely by specialised equipment. To simplify the model, we notice that, upon surface reflection, light is mainly linearly polarised. As a consequence, we can model the three polarisation components including the unpolarised intensity, the phase of linear polarisation and the degree of polarisation, instead of four elements of the Stokes vector.

The theoretical contribution of this paper is a model of polarisation upon specular reflection from rough surfaces. The model is simpler than the one proposed in [11]. Despite having less degrees of freedom, our model can describe the influence of the surface geometry and physical parameters such as the micro-facet structure and the refractive index on specular polarisation. Departed from the statistical model of surface microfacets in [6], [10], we derive the polarisation components of the electric fields that are perpendicular and parallel to the plane of reflection, i.e. the plane spanned by the surface normal and the viewer direction. In the model, the polarisation components depend on the index of refraction, the angle of incidence and the surface roughness. To our knowledge, no other work 
has provided a model which relates polarisation to microfacet structure based on the Fresnel reflection theory.

Furthermore, we demonstrate the application of the model to the simulation of a polarising filter using only a single unpolarised image. This approach confers advantages in emulating polarisation effects without the use of a polariser. In this respect, our theory offers a software-based alternative to optical polarising filters used in photography. This is particularly useful and beneficial for cameras with no mounting threads for polarisers, including camera phones and compact point-and-shoot cameras. The resulting polarisation imagery will facilitate further applications such as metal/dielectric classification [13], recognition of camouflaged objects, glare reduction and dynamic range enhancement.

The paper is structured as follows. In Section II, we propose a polarisation model for rough surfaces. Subsequently, we make use of this model to reconstruct the minimal and maximal polarisation components from a single unpolarised image in Section III. The reconstruction of polarisation components takes into account the surface geometry, the surface roughness and the material refractive index. In Section IV, we presents results on estimating polarisation components and simulating a polarising filter, followed by our conclusion.

\section{Polarisation Model for Rough Surfaces}

We commence by introducing polarisation images and polarisation components in Section II-A. Subsequently, in Section II-B, we present a micro-facet distribution model in a local coordinate system related to the viewer direction, the surface normal and the microfacet normal. This allows us to model the polarisation mechanism upon reflection from rough surfaces and to relate the polarisation components to the diffuse and specular components of an unpolarised image in Section II-C.

\section{A. Polarisation Images}

A polarisation image exhibits the transmitted radiance of the electric field vector oscillating along a certain orientation in the plane perpendicular to the direction of propagation. This phenomenon can be measured by mounting a linear polariser in front of the camera's optics. By rotating the polariser, one can capture polarisation components that are oriented at different angles in a plane orthogonal to the light propagation direction. With this setup, the intensity captured at each image pixel varies sinusoidally with respect to the angle of rotation of the polariser. According to this model, the intensity $I$ fluctuates along a Transmitted Radiance Sinusoid (TRS) curve bounded by $I_{\min }$ and $I_{\max }$. Let $\vartheta$ be the polariser orientation angle with respect to a reference axis and $\psi$ be the phase angle of the sinusoid. Also, we denote $\lambda$ as the sampled wavelength of the image and $u$ as the pixel index. With this notation, the intensity of the polarisation component corresponding to the orientation $\vartheta$ is expressed as

$$
I(u, \lambda, \vartheta)=\frac{I_{\max }+I_{\min }}{2}+\frac{I_{\max }-I_{\min }}{2} \cos (2 \vartheta-2 \psi)
$$

Here, for brevity reasons, we have omitted the pixel and wavelength indices in the notations of $I_{\min }, I_{\max }$ and the phase $\psi$.

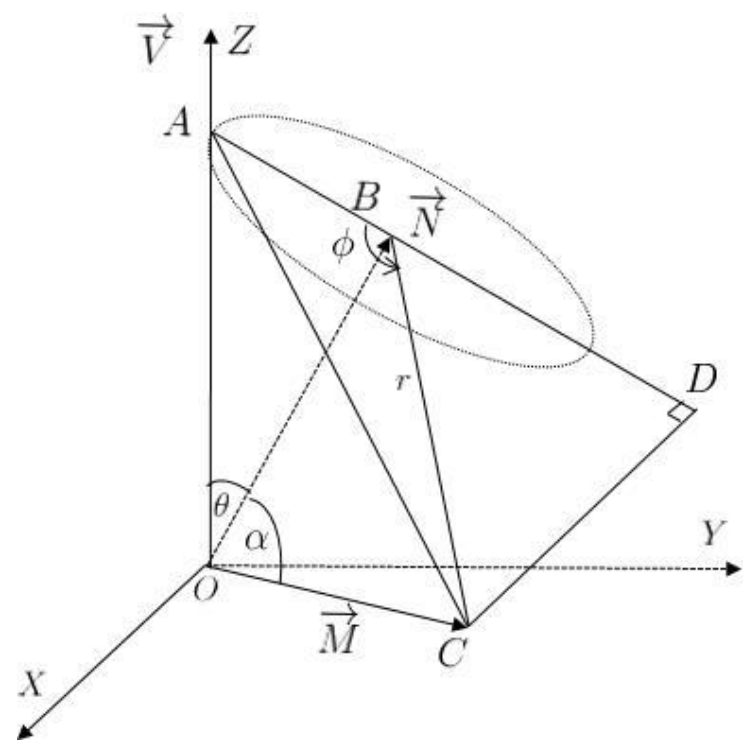

Fig. 1. Geometry of the micro-facet distribution.

\section{B. Micro-facet Distribution Model}

In [6], surfaces with microscopic level of detail can be modelled using a statisically large collection of mirror-like microfacets. Light arriving at these surfaces either reflects off a microfacet a single time, or undergoes multiple specular reflections from the microfacets, or penetrates into and scatters within the material body before being refracted back into the air. The first phenomenon accounts for surface specular reflection while the latter two accounts for diffuse reflection. Similar to the work in [13], we assume that the diffuse reflection component is unpolarized due to the random nature of multiple reflections and scattering whereas the specular component causes partial polarisation according to the Fresnel law of reflection.

The coordinate system used throughout the paper is depicted in Figure 1. Here, the origin is the surface location $O$ under observation and the $z$-axis is aligned to the viewer direction $\vec{V}$. In this coordinate system, we view the object surface as being composed of microfacets, each with a normal direction $\vec{M}$. The average slope of these microfacets can be represented by the mean surface normal $\vec{N}$ with unit length, i.e. $\|\vec{N}\|=1$, which lies on the $y z$-plane spanned by the viewer's direction $\vec{V}$ and the $y$-axis.

Note that the unit surface normal $\vec{N}$ with the terminal point $B$ forms an angle $\theta$ with the viewer direction $\vec{V}$. Since we only need to consider surface points visible to the viewer, we have $\theta \leq \frac{\pi}{2}$. Moreover, we suppose that the plane passing through $B$ and perpendicular to $\vec{N}$ intersects the $z$-axis at $A$ and the microfacet normal $\vec{M}$ at $C$. We choose to parameterise the microfacet normal via the location of $C$. In fact, the position of this point is uniquely determined by the angle $\phi$ between the vector $\overrightarrow{B A}$ and $\overrightarrow{B C}$ and the distance $r$ between the points $B$ and $C$.

Now we consider a statistical model for the distribution of the microfacet normal about the mean surface normal. 


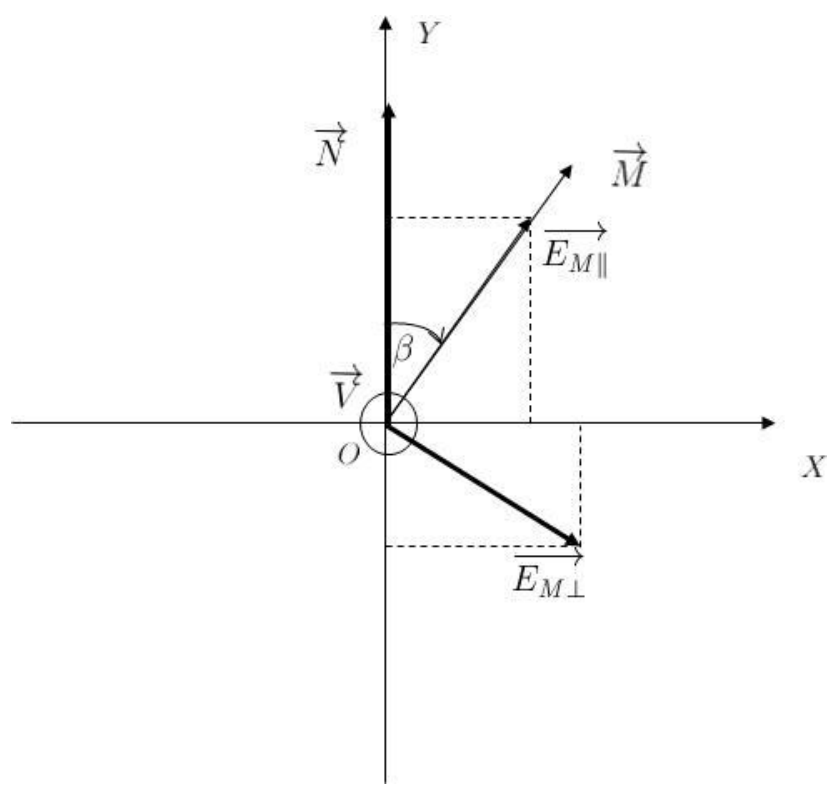

Fig. 2. A cross section of the decomposition of the reflected electromagnetic field observed from the viewer's position.

Similar to the work in [6], we assume that the surface under consideration is isotropic and adopt a Gaussian model for the microfacet normal distribution. In effect, the angle $\alpha$ between the facet normal $\vec{M}$ and the mean surface normal $\vec{N}$ assumes a Gaussian distribution with a zero mean. Therefore, the probability distribution of the microfacet normal direction is given by

$$
\begin{aligned}
P(\vec{M}) & =K \exp \left(-\sigma \tan ^{2} \alpha\right) \\
& =K \exp \left(-\sigma r^{2}\right)
\end{aligned}
$$

since, from Figure 1, it is straightforward to deduce that $\tan \alpha=r$. Here, $K$ is the normalising constant of the microfacet distribution, $\sigma$ indicates the roughness of the microfacet structure.

\section{Polarisation Upon Reflection from Rough Surfaces}

In this section, we derive a polarimetric reflection model for rough surfaces. To achieve this, we consider the electric field reflected from each microfacet and integrate the power of these fields across all the possible microfacet orientations. The polarisation effect is modelled by decomposing these fields into two orthogonal components, one of which lies in the mean plane of reflection, which is formed by the mean surface normal $\vec{N}$ and the viewing direction $\vec{V}$ in Figure 1 , and the other one perpendicular to this plane.

Let us consider a microfacet with the normal $\vec{M}$. As light is reflected by this facet in the viewer direction $\vec{V}$ (the $z$ axis), it induces an electromagnetic field with the electric field vector oscillating about and perpendicular to $\vec{V}$. In Figure 2, we show a cross-section of Figure 1 as observed from the viewer's position so as to illustrate the electric field reflected from the microfacet, where the mean surface normal $\vec{N}$ is aligned with the $y$ axis.

In the figure, the electric field vector of the reflected light is decomposed into two orthogonal harmonic components. One of the components, i.e. $\overrightarrow{E_{M \|}}$ is in the plane of reflection formed by the vectors $\vec{V}$ and $\vec{M}$ while the other one, i.e. $\overrightarrow{E_{M \perp}}$ is perpendicular to this plane. These two components propagate within their respective planes with their magnitudes varying according to a sinusoidal pattern.

Now we formulate the radiance power of each of the two components above. In the local coordinate system, let $D$ be the orthogonal projection of $C$ onto the $x y$ plane. Since the plane passing through $A, B$ and $C$ is perpendicular to the $x y$ plane and they intersect across the line going through $A$ and $B, D$ belongs to this line. Therefore, the magnitudes of the vectors spanned by $A, B$ and $D$ are related as

$$
\|\overrightarrow{A D}\|=\|\overrightarrow{A B}\|+\|\overrightarrow{B D}\|=\tan \theta-r \cos \phi
$$

Therefore

$$
\begin{aligned}
& \overrightarrow{A D}=\frac{\|\overrightarrow{A D}\|}{\|\overrightarrow{A B}\|} \overrightarrow{A B} \\
& =[0, \sin \theta-r \cos \theta \cos \phi,-\sin \theta \tan \theta+r \sin \theta \cos \phi]
\end{aligned}
$$

As a result, $\overrightarrow{O D}=\overrightarrow{O A}+\overrightarrow{A D}=[0, \sin \theta-$ $r \cos \theta \cos \phi, \cos \theta+r \sin \theta \cos \phi]$, and $\vec{M}=\overrightarrow{O C}=$ $[r \sin \phi, \sin \theta-r \cos \theta \cos \phi, \cos \theta+r \sin \theta \cos \phi]$.

Let $\beta$ be the angle between the reflection plane for the microfacet, i.e. the plane formed by the vectors $\vec{V}$ and $\vec{M}$ and the mean plane of reflection formed by the vectors $\vec{V}$ and $\vec{N}$. As illustrated in Figure 2, it is also the angle between the positive $y$-axis and the projection of $\vec{M}$ onto the $x y$-plane, whose coordinates are $[r \sin \phi, \sin \theta-r \cos \theta \cos \phi, 0]$. Thus, we can write

$$
\cos \beta=\frac{\sin \theta-r \cos \theta \cos \phi}{\sqrt{r^{2} \sin ^{2} \phi+(\sin \theta-r \cos \theta \cos \phi)^{2}}}
$$

Similarly, let $\gamma$ be the reflection angle for the microfacet, i.e. the angle between $\vec{M}$ and $\vec{V}$. This angle is related to the geometric variables through the expression

$$
\cos \gamma=\frac{\cos \theta+r \sin \theta \cos \phi}{\sqrt{r^{2}+1}}
$$

We can safely assume that polarisation upon reflection is mainly contributed by incident light rays lying in the microfacet plane of reflection, such that the microfacet normal $\vec{M}$ is the bi-sector of the incident direction and the view direction $\vec{V}$. Since we can further assume that the incident light is uniformly distributed in all the incoming directions, we denote the intensity of the incident light rays as a constant $I_{i}$.

According to the Fresnel reflection theory [14], the intensities $J_{\|}(M)$ and $J_{\perp}(M)$ of the polarisation components $\overrightarrow{E_{M \|}}$ and $\overrightarrow{E_{M \perp}}$ are given by

$$
\begin{aligned}
J_{\|}(M) & =F_{\|}(\gamma, \eta) I_{i} \\
J_{\perp}(M) & =F_{\perp}(\gamma, \eta) I_{i}
\end{aligned}
$$

where $F_{\|}(\gamma, \eta)$ and $F_{\perp}(\gamma, n)$ are the Fresnel parallel and perpendicular reflection coefficients, respectively, and $\eta$ is the material refractive index. 
To quantify the polarisation effect contributed by the microfacet, we project the power of the components $\overrightarrow{E_{M \|}}$ and $\overrightarrow{E_{M \perp}}$ onto the mean plane of reflection (the $y z$-plane) and the plane perpendicular to the former one (the $x z$-plane). Since $\beta$ is the angle between $\overrightarrow{E_{M \|}}$ and the mean surface normal $\vec{N}$, the radiance $I_{\|}(M)$ and $I_{\perp}(M)$ of the parallel and perpendicular components contributed by the microfacet with respect to the mean plane of reflection can be derived by Malus's law [15] as

$$
\begin{aligned}
I_{\|}(M) & =J_{\|}(M) \cos ^{2} \beta+J_{\perp}(M) \sin ^{2} \beta \\
I_{\perp}(M) & =J_{\perp}(M) \cos ^{2} \beta+J_{\|}(M) \sin ^{2} \beta
\end{aligned}
$$

Lastly, to quantify the overall effect of polarisation contributed by all the microfacets, we integrate the intensities of the parallel and perpendicular components they induce with respect plane of reflection subject to the microfacet normal distribution in Equation 2

$$
\begin{aligned}
I_{\|}= & \int I_{\|}(M) P(\vec{M}) d \vec{M} \\
= & K I_{i} \times \int_{0}^{2 \pi} \int_{0}^{\infty}\left(\left(F_{\|}(\gamma, \eta) \cos ^{2} \beta\right.\right. \\
& \left.\quad+F_{\perp}(\gamma, \eta) \sin ^{2} \beta\right) \exp \left(-\sigma r^{2}\right) d r d \phi \\
I_{\perp}= & \int I_{\perp}(M) P(\vec{M}) d \vec{M} \\
= & K I_{i} \times \int_{0}^{2 \pi} \int_{0}^{\infty}\left(F_{\perp}(\gamma, \eta) \cos ^{2} \beta\right. \\
& \left.\quad+F_{\|}(\gamma, \eta) \sin ^{2} \beta\right) \exp \left(-\sigma r^{2}\right) d r d \phi
\end{aligned}
$$

In Equations 5 and $6, I_{\|}$and $I_{\perp}$ are the overall magnitudes of the polarisation components parallel and perpendicular to the mean plane of reflection.

\section{Polarisation Component Reconstruction}

With the polarimetric reflection model above, we aim to reconstruct the polarisation component at an arbitrary polariser angle $\vartheta$. Here, we assume that the scene geometry and photometric variables including the material refractive index $\eta$ and the surface roughness $\sigma$ have been recovered at every pixel and wavelength using an approach akin to those in [16], [17]. Further, suppose that the input unpolarised radiance image has been decomposed into a diffuse and a specular reflection components with intensities $I_{d}$ and $I_{s}$. Such a decomposition on a single image can be effected by well known methods using the maximum chromaticity intensity space [18] or partial differential equations [19]. As light is polarised upon specular reflection, we observe that the transmitted radiance is maximal when the polariser orientation is perpendicular to the plane of reflection and minimal when it is parallel to the plane of reflection. Based on the principle of linear superposition, the sum of the magnitudes of the perpendicular and parallel components of polarisation must be equal to the specular component. In other words

$$
I_{s}=I_{\perp}+I_{\|}
$$

From Equations 5 and 6, we note that the ratio $R \triangleq \frac{I_{\|}}{I_{\perp}}$ is a function of the reflection angle $\theta$, the surface roughness $\sigma$ and the material refractive index $\eta$

$$
R=\frac{\int_{0}^{2 \pi} \int_{0}^{\infty}\left(F_{\|}(\gamma, \eta) \cos ^{2} \beta+F_{\perp}(\gamma, \eta) \sin ^{2} \beta\right) \exp \left(-\sigma r^{2}\right) d r d \phi}{\int_{0}^{2 \pi} \int_{0}^{\infty}\left(F_{\perp}(\gamma, \eta) \cos ^{2} \beta+F_{\|}(\gamma, \eta) \sin ^{2} \beta\right) \exp \left(-\sigma r^{2}\right) d r d \phi}
$$

This ratio can be pre-computed by numerical integration once the above parameters are known through a look-up table. This permits the recovery of the perpendicular and parallel components through the expressions

$$
\begin{aligned}
I_{\perp} & =\frac{1}{1+R} I_{s} \\
I_{\|} & =\frac{R}{1+R} I_{s}
\end{aligned}
$$

Moreover, the transmitted radiance along the Transmitted Radiance Sinusoid consists of both the unpolarised and partially polarised components of the reflected light. Using a similar formulation to that in [13], we reconstruct the polarimetric components $I_{\max }$ and $I_{\min }$ using the following relations

$$
\begin{aligned}
& I_{\max }=\frac{I_{d}}{2}+I_{\perp} \\
& I_{\min }=\frac{I_{d}}{2}+I_{\|}
\end{aligned}
$$

In Equations 9 and 10, we note that the polarisation effects are caused by specular reflection. By a similar derivation to that in [20], the phase angle $\psi(u)$ at a pixel $u$ is related to the azimuth angle $\varphi(u)$ of the surface normal at the same location as $\psi(u)=\varphi(u)$ or $\psi(u)=\varphi(u)+\pi$. With the polarisation components $I_{\max }, I_{\min }$ and $\psi$ at hand, we can reconstruct the polarisation component oriented at an arbitrary angle $\vartheta$ using Equation 1. Since the TRS is a periodical function with a period of 180 degrees, it is invariant to the choice of the phase angle $\psi(u)$ between $\varphi(u)$ and $\varphi(u)+\pi$.

\section{EXPERIMENTS}

In this section, we present experiments on the recovery of the polarimetric components and the simulation of a polarising filter. To this end, we employ a synthetic dataset and two datasets of real-world imagery. The use of synthetic data allows quantitative analysis where the accuracy of the recovered polarisation component can be compared to the ground-truth. On the other hand, the use of real-world images serves to illustrate the utility of our model for the simulation of a virtual polariser on unpolarised images.

To generate the synthetic dataset, we render these synthetic surfaces with the refractive index of the 24 plastic and liquid materials reported in [21], and the empirical measurements of the surface roughness of carbon, rubber obsidian, olive drab and rust reported in [22]. The polarisation images were rendered according to the Torrance-Sparrow model [6]. For each combination, ground truth polarisation images were generated for five polariser orientations at $0,30,45,60$ and 90 degrees in the anti-clockwise direction with respect to the vertical axis of the image coordinate system. In Figure 3, we show samples of the unpolarised synthetic images generated. 

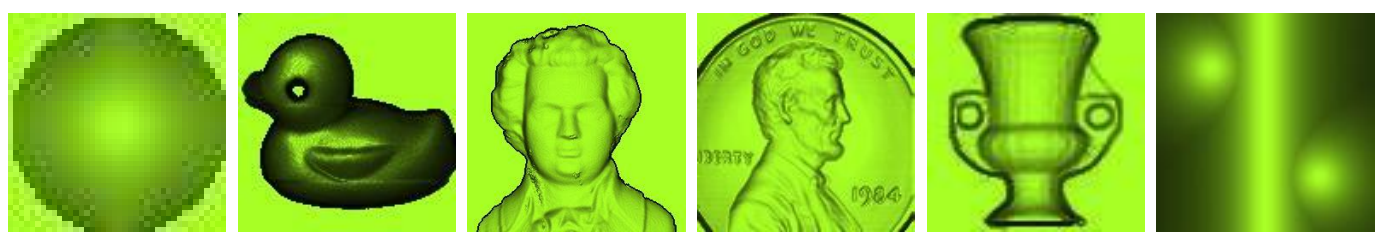

Fig. 3. Samples of unpolarised synthetic images.
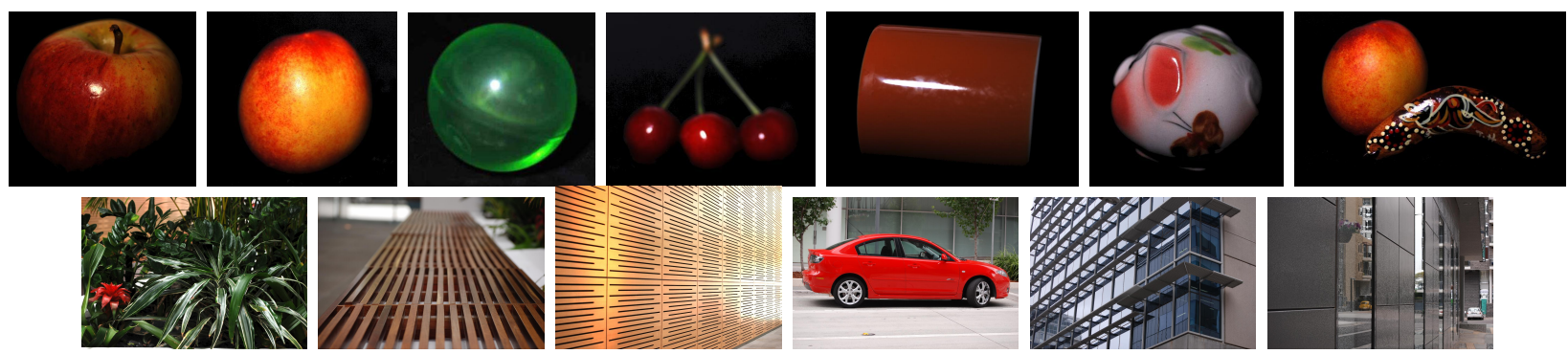

Fig. 4. Unpolarised imagery for the Indoor (top row) and the Outdoor dataset (bottom row).

Of the real-world imagery, the first dataset comprises images of diverse objects, i.e. ceramics, fruits and plastic items, acquired in-house under artificial sun lights. Here, we denote this as the Indoor dataset. The second real-world dataset, which we denote the Outdoor dataset, corresponds to outdoor scenes containing a strong partially polarised component. In Figure 4 we show sample views of each of the objects and outdoor scenes in our real-world datasets. To acquire our imagery, we have used a Nikon D80 camera which is able to give highresolution images of up to 10 Mega pixels. Each of the images has been acquired using a linear polariser mounted in front of the lens. The polariser is multi-coated to minimise internal reflection and glare within the optics, and is assumed to have uniform transmission over its surface. To acquire polarisation imagery, we rotate the polariser such that its transmission axis orientation is incremented in steps of 30 degrees, to form angles between 0 and 150 degrees with respect to the vertical axis in the image plane.

As presented earlier, to evaluate the ratio in Equation 8, we require the surface normals, the refractive index and the surface roughness to be recovered at every pixel and wavelength of the real-world images. To this end, we first estimate the image reflectance by normalising the input image by the illumination colour obtained through a method such as Gray World [23]. Subsequently, we employ the method in [17] to recover the scene geometry and photometric parameters simultaneously from a single image. To obtain the diffuse and specular components for the recovery of $I_{\max }$ and $I_{\min }$ through Equations 11 and 12, we apply the method of Tan et al. [18] to the image under consideration. In addition, the phase angle of polarisation can be deduced from the azimuth angle of the recovered surface normals. Having obtained these three polarisation components, the polarisation intensity can be evaluated at arbitrary polariser angles.

\section{A. Polarisation Component Estimation}

We commence by evaluating the errors of the generated polarimetric components for the synthetic dataset. These errors
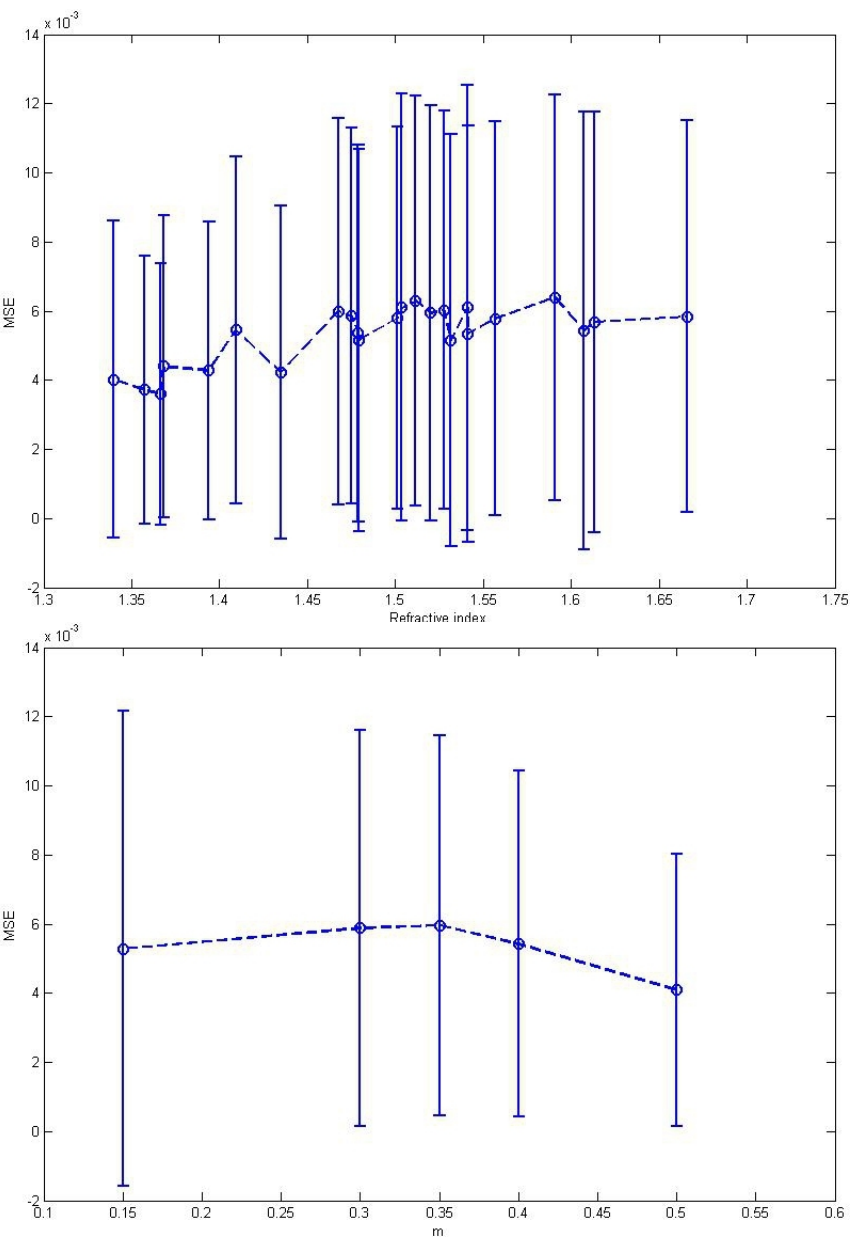

Fig. 5. Normalised mean squared error of estimating the polarisation components from the synthetic dataset. Top panel: The average error across the dataset with respect to the refractive index. Bottom panel: The average error across the dataset with respect to the surface roughness. 

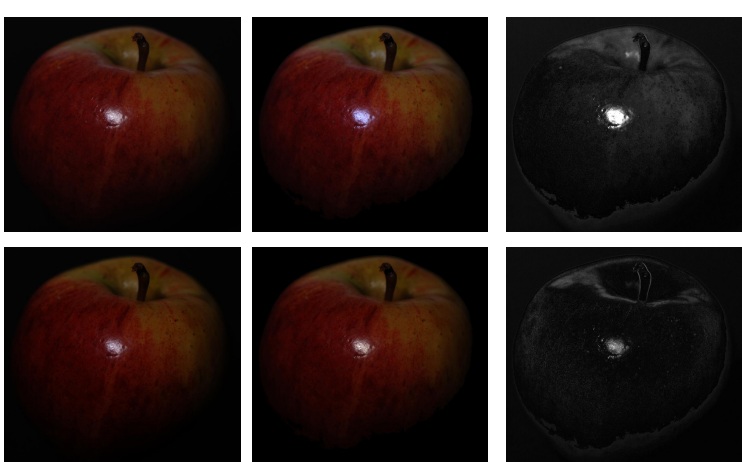

Ground-truth

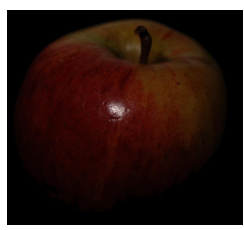

Reconstruction
Colour difference

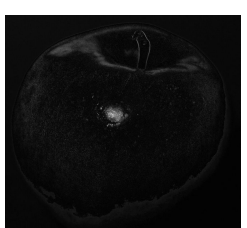

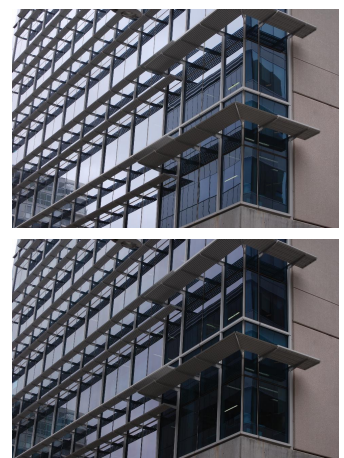

Ground-truth

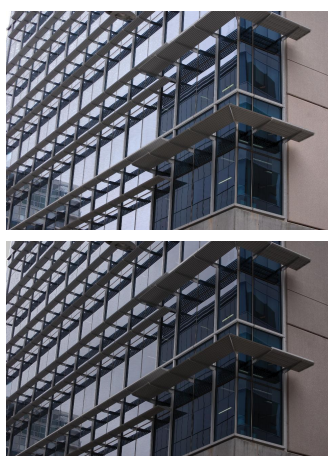

Reconstruction

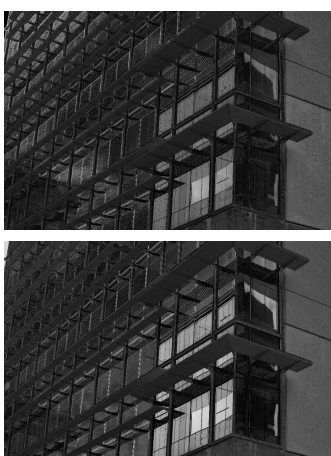

Colour difference

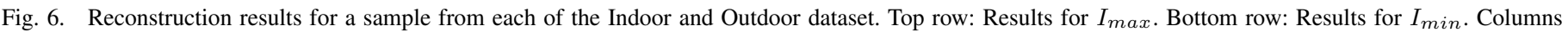

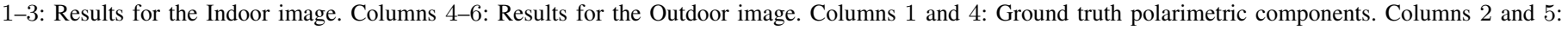
Reconstructed polarimetric component. Columns 3 and 6: The normalised colour difference map.

\begin{tabular}{|l|c|c|c|c|}
\hline & $I_{\max }(\Delta E)$ & $I_{\max }(\mathrm{MSE})$ & $I_{\min }(\Delta E)$ & $I_{\min }(\mathrm{MSE})$ \\
\hline Indoor Dataset & $2.09 \pm 0.64$ & $0.008 \pm 0.0011$ & $1.24 \pm 0.31$ & $0.0040 \pm 0.0011$ \\
\hline Outdoor Dataset & $4.40 \pm 1.95$ & $0.0716 \pm 0.0473$ & $4.44 \pm 2.26$ & $0.0406 \pm 0.0418$ \\
\hline
\end{tabular}

TABLE I. THE ERROR OF THE RECOVERED $I_{\max }$ AND $I_{\min }$ AVERAGED ACROSS IMAGE PIXELS IN THE TWO DATASETS. THE FIRST AND THIRD COLUMNS REPORT THE COLOUR ERROR IN TERMS OF “JUST NOTICEABLE DIFFERENCE” (JND), WHEREAS THE SECOND AND FOURTH COLUMNS REPORT THE NORMALISED MEAN SQUARED ERROR (MSE)

were measured as the mean squared error (MSE) difference between the normalised reconstructed and ground truth polarisation components at the orientations of $0,30,45,60$ and 90 degrees. In Figure 5, we report the mean and standard derviation of the MSE error with respect to the material refractive index in the top panel and the surface roughness in the bottom panel. Overall, the error only varies slightly between different material refractive indices between $4 \times 10^{-3}$ and $6 \times 10^{-3}$. Similarly, it does not change significantly across different surface roughness levels as shown in the bottom panel of Figure 5. Note that the results shown here implies that our method can sustain a stable error margin for various photometric variables including the refractive index and surface roughness.

Next, we present results on the recovery of the polarimetric components $I_{\max }$ and $I_{\min }$. As described above, these components are independent of the polariser orientation. To quantify the error of the estimated components, we have used the CIE Delta $\mathrm{E}(\Delta E)$ colour distance to measure the difference between the polarimetric components recovered by our method and the ground truth images. The $\Delta E$ is a perceptual metric which aims at measuring the "just noticeable difference" (JND) between colour pairs in the CIELab colour space. Note that, in the CIE76 standard used here, a unit of JND corresponds to a $\Delta E \approx 2.3$ [24].

In Figure 6, we present sample qualitative results of the $I_{\max }$ and $I_{\min }$ components for an object in the Indoor dataset and a scene in the Outdoor one. In the first three columns of the figure, we show the results for the Indoor image whereas in the last three, we show the results for the Outdoor scene. In the left-hand columns of the figures, we show the ground truth polarimetric components of the Indoor image. Our estimates for the Indoor image are shown in the second columns whereas the corresponding error maps, where black accounts for no error and white is given by the maximum absolute difference between the ground truth and our estimate, are shown in the right-hand columns. Note that the error map has been scaled to allow for better visual inspection, such that pure white corresponds to $10 \mathrm{JND}$ units. In general, the constructed $I_{\max }$ is brighter than $I_{\min }$ as shown in the figures. In the last three columns of Figure 6, we note that the reconstructed $I_{\text {min }}$ component is almost free of specular reflection while the constructed $I_{\max }$ exhibits a significant amount of specularity, which resembles the ability of an actual polariser to remove specularities.

In Table I, we show the average per-pixel $\Delta E$ difference and the normalised mean squared error (MSE) for the two datasets. For the Indoor dataset, the $\Delta E$ difference is well within the JND, with an average of 2.09 for $I_{\max }$ and 1.24 for $I_{m i n}$. For the Outdoor scenes, it is on average 4.4 for $I_{\max }$ and 4.44 for $I_{\min }$, i.e. less than two JND. Note that this error metric is a distance measure in the $L a b$ color space and the degree of error shown here is quite small compared to the range of the $L a b$ coordinates. This implies that our recovered polarimetric components are, on average, indistinguishable from the ground truth for the average human observer. In addition, the mean squared errors after the input image intensities are normalised to the range $[0,1]$ are quite small. For the indoor images, these are 0.008 and 0.004 for $I_{\max }$ and $I_{\min }$ respectively. The corresponding errors for the outdoor images are 0.0716 and 0.0406 for $I_{\max }$ and $I_{\min }$. Since the MSE and $\Delta E$ errors are correlated, the low MSE for the outdoor and indoor images also implies a low $\Delta E$ error, which renders the colour error indistinguishable to the human eye.

\section{B. Simulating a Polarising Filter}

We now turn our attention to the simulation of a polarising filter from a single unpolarised image. Note that, in contrast with the experiments above, in the case of a "virtual" polariser, 

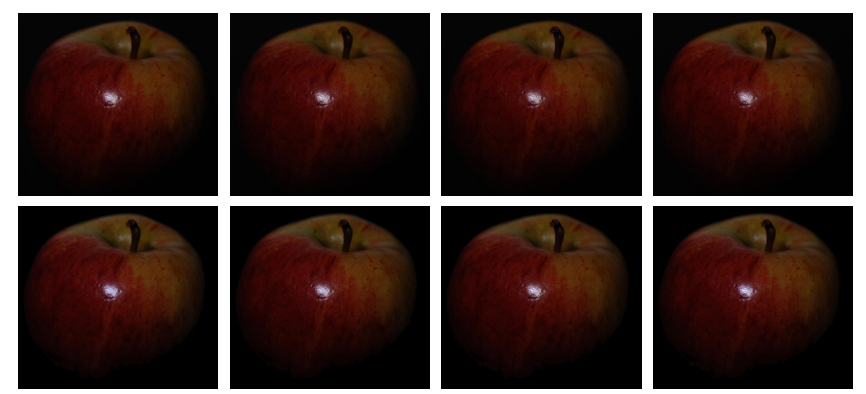

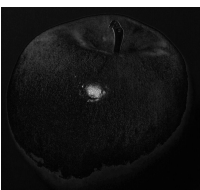

$0^{\circ}$

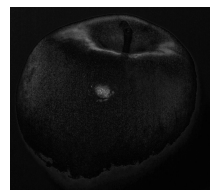

$30^{\circ}$

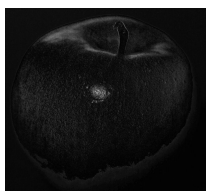

$60^{\circ}$

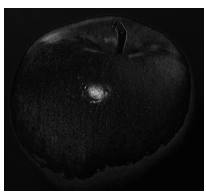

$90^{\circ}$
Fig. 7. A simulation of the polarisation components of an indoor scene. First row: ground-truth polarisation images. Second row: reconstructed polarisation images corresponding to the polarisation angles shown. Third row: The normalised colour difference map.
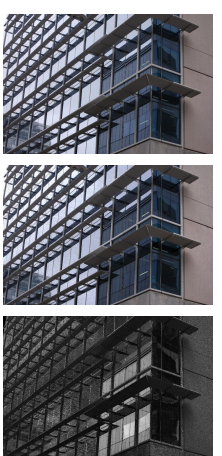

$0^{\circ}$
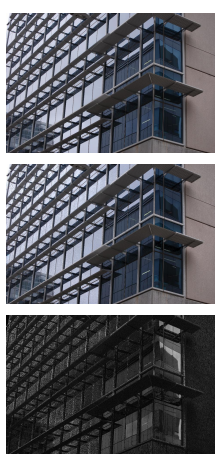

$30^{\circ}$
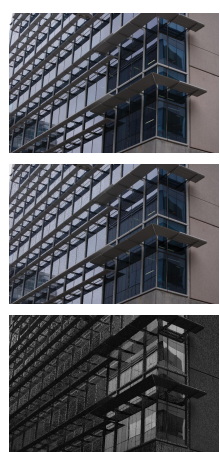

$60^{\circ}$
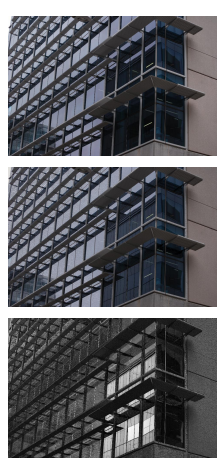

$90^{\circ}$
Fig. 8. A simulation of the polarisation components of an outdoor scene. First row: ground-truth polarisation images. Second row: reconstructed polarisation images corresponding to the polarisation angles shown. Third row: The normalised colour difference map.

the synthesised imagery will be a function of the polariser orientation angle $\vartheta$ as given in Equation 1. As before, here we measure the difference between the simulated imagery and the corresponding ground truth images captured with our linear polariser in terms of the quantity $\Delta E$ as a function of $\vartheta$.

In Figures 7 and 8 we show the simulated imagery for two sample images used for our previous experiments. In the figures, we show from top-to-bottom, the ground truth, simulated images and normalised difference map in increasing polariser orientation angles $\vartheta=\left\{0^{\circ}, 30^{\circ}, 60^{\circ}, 90^{\circ}\right\}$ from left to right. Again, the error map has been scaled in the same manner as for $I_{\min }$ and $I_{\max }$ for visualisation purposes. We note the shift in specularity in the synthetic images with respect to the polariser orientation angle.

Finally, we show in Figure 9 the $\Delta E$ difference as a function of the polariser orientation angle $\vartheta$ for the two datasets. Here, the error for the outdoor dataset is higher than the indoor one due to the geometric complexity in the outdoor scenes. The errors presented here are, again, consistent with our previous results, where the $\Delta E$ for the polarimetric
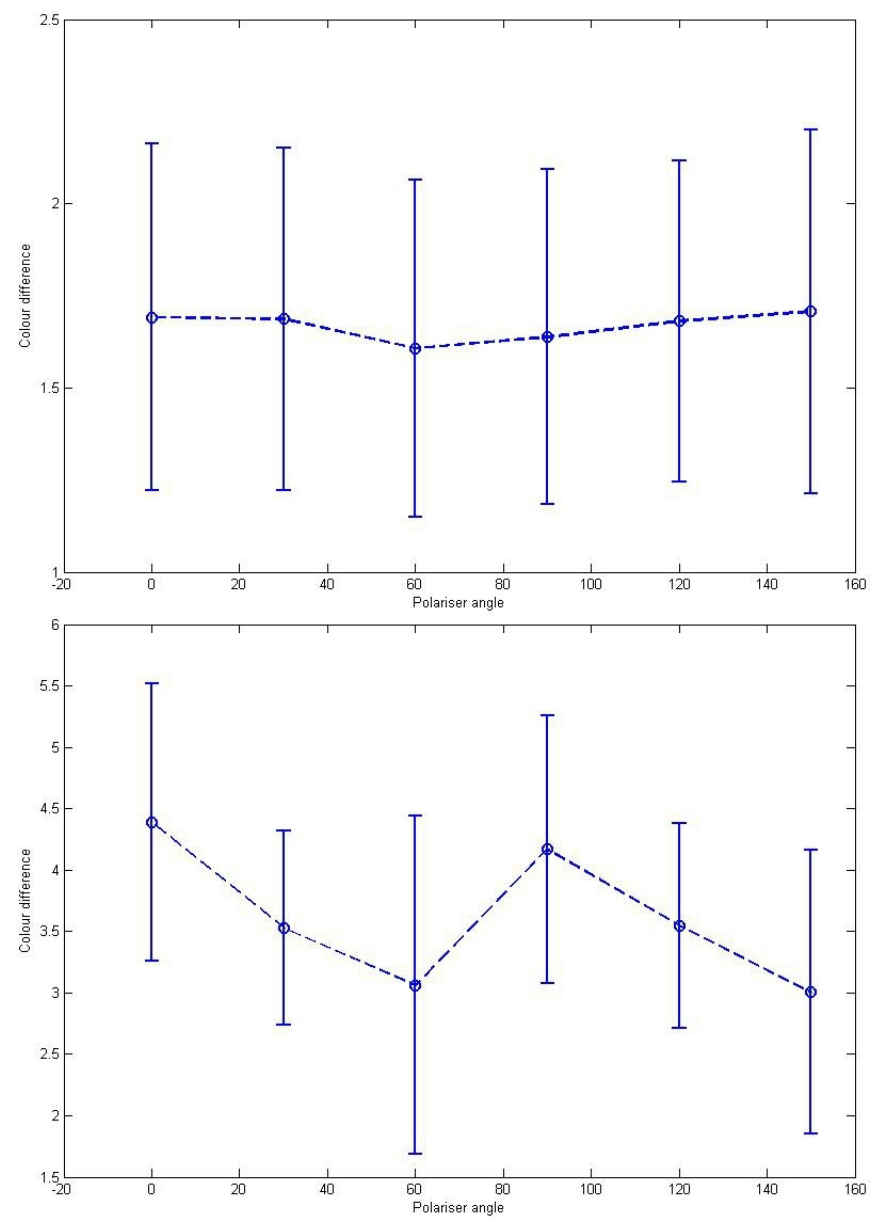

Fig. 9. Per-pixel average colour difference $(\Delta E)$ with respect to the polariser orientation angle $\vartheta$. Top panel: Results for the Indoor dataset. Bottom panel: Results for the Outdoor dataset.

components is on average, within the JND for the indoor dataset and below two JND for the outdoor dataset. In other words, these numerical errors between the ground truth and the simulated imagery are barely noticeable to a human observer.

\section{CONCLUSIONS}

In this paper, we have developed a model for reconstructing polarisation components from a single unpolarised imagery. We have shown how the maximal and minimal polarimetric intensities can be formulated as a function of the refractive index, the surface roughness and the surface normal. Combining a model of the microfacet structure of rough surfaces with the Fresnel reflection theory, we reconstruct the polarimetric components. This work is not only theoretically important but also practically useful since it provides a link between the microfacet structure and polarisation based on the Fresnel theory. The presented work has potential applications in simulating a polarising filter on real-world imagery.

\section{ACKNOWLEDGMENT}

NICTA is funded by the Australian Government as represented by the Department of Broadband, Communications and the Digital Economy and the Australian Research Council through the ICT Centre of Excellence program. 


\section{REFERENCES}

[1] L. B. Wolff, "Polarization vision: a new sensory approach to image understanding," Image Vision Computing, vol. 15, no. 2, pp. 81-93, 1997.

[2] F. Goudail, P. Terrier, Y. Takakura, L. Bigué, F. Galland, and V. DeVlaminck, "Target detection with a liquid-crystal-based passive stokes polarimeter," Applied Optics, vol. 43, no. 2, pp. 274-282, Jan 2004.

[3] L. B. Wolff and T. E. Boult, "Polarization/Radiometric Based Material Classification," in Computer Vision and Pattern Recognition, 1989, pp. 387-395.

[4] H. Chen and L. Wolff, "Polarization phase based method for material classification in computer vision," IJCV, vol. 28, no. 1, pp. 73-83, June 1998.

[5] M. Oren and S. K. Nayar, "Generalization of the Lambertian Model and Implications for Machine Vision," International Journal of Computer Vision, vol. 14, no. 3, pp. 227-251, April 1995.

[6] K. Torrance and E. Sparrow, "Theory for Off-Specular Reflection From Roughened Surfaces," Journal of the Optical Society of America, vol. 57, no. 9, pp. 1105-1112, Sep 1967.

[7] P. Beckmann and A. Spizzichino, The Scattering of Electromagnetic Waves from rough Surfaces. Pergamon Press, 1963.

[8] L. Wolff and T. Boult, "Constraining object features using a polarization reflectance model," IEEE Transactions on Pattern Analysis and Machine Intelligence, vol. 13, no. 7, pp. 635-657, 1991.

[9] L. B. Wolff, "Diffuse-reflectance model for smooth dielectric surfaces," Journal of the Optical Society of America, vol. 11, no. 11, pp. 29562968, November 1994.

[10] K. E. Torrance, E. M. Sparrow, and R. C. Birkebak, "Polarization, Directional Distribution, and Off-Specular Peak Phenomena in Light Reflected from Roughened Surfaces," Journal of the Optical Society of America, vol. 56, no. 7, pp. 916-924, Jul. 1966.

[11] R. G. Priest and T. A. Germer, "Polarimetric brdf in the microfacet model: Theory and measurements," in Proceedings of the 2000 Meeting of the Military Sensing Symposia Specialty Group on Passive Sensors, vol. 1, 2000, pp. 169-181.

[12] V. Thilak, D. G. Voelz, and C. D. Creusere, "Polarization-based index of refraction and reflection angle estimation for remote sensing applications," Applied Optics, vol. 46, no. 30, pp. 7527-7536, Oct 2007.

[13] L. B. Wolff, "Polarization-based material classification from specular reflection," IEEE Transactions on Pattern Analysis and Machine Intelligence, vol. 12, no. 11, pp. 1059-1071, 1990.

[14] M. Born and E. Wolf, Principles of Optics: Electromagnetic Theory of Propagation, Interference and Diffraction of Light ( $7^{\text {th }}$ Edition), 7th ed. Cambridge University Press, 1999.

[15] E. Hecht, Optics, 4th ed. Addison-Wesley, 2002.

[16] K. Hara, K. Nishino, and K. Ikeuchi, "Light Source Position and Reflectance Estimation from a Single View without the Distant Illumination Assumption," IEEE Transactions on Pattern Analysis and Machine Intelligence, vol. 27, pp. 493-505, April 2005.

[17] C. P. Huynh and A. Robles-Kelly, "Simultaneous Photometric Invariance and Shape Recovery," in International Conference on Computer Vision, 2009, pp. 1757-1764.

[18] R. T. Tan and K. Ikeuchi, "Separating reflection components of textured surfaces using a single image," IEEE Trans. Pattern Anal. Mach. Intell., vol. 27, no. 2, pp. 178-193, February 2005.

[19] S. P. Mallick, T. Zickler, P. N. Belhumeur, and D. J. Kriegman, "Specularity Removal in Images and Videos: A PDE Approach," in $\operatorname{ECCV}(1), 2006$, pp. 550-563.

[20] G. A. Atkinson and E. R. Hancock, "Shape estimation using polarization and shading from two views," IEEE Trans. Pattern Anal. Mach. Intell. vol. 29, no. 11, pp. 2001-2017, 2007.

[21] S. N. Kasarova, N. G. Sultanova, C. D. Ivanov, and I. D. Nikolov, "Analysis of the dispersion of optical plastic materials," Optical Materials, vol. 29, no. 11, pp. 1481 - 1490, 2007.

[22] R. L. Cook and K. E. Torrance, "A reflectance model for computer graphics," ACM Transactions on Graphics, vol. 1, no. 1, pp. 7-24, Jan 1982.
[23] G. Buchsbaum, "A spatial processor model for object colour perception," Journal of the Franklin Institute, vol. 310, no. 1, pp. 1 - 26, 1980.

[24] G. Sharma, Digital Color Imaging Handbook. CRC Press, 2003. 